\title{
ON THE PARTIAL SUMS OF FOURIER SERIES AT POINTS OF DISCONTINUITY
}

\author{
BY \\ OTTO SZÁSZ
}

1. Introduction. Consider a Fourier sine series

$$
f(\theta) \sim \sum_{1}^{\infty} b_{\nu} \sin \nu \theta, \quad b_{\nu}=(2 / \pi) \int_{0}^{\pi} f(\theta) \sin \nu \theta d \theta, \quad 0<\theta<\pi,
$$

and write

$$
s_{n}(\theta)=\sum_{1}^{n} b_{\nu} \sin \nu \theta, \quad n=1,2,3, \cdots .
$$

Fejér proved (cf. Zygmund [5, p. 181]) ${ }^{(1)}$ that if $f(\theta)$ is of bounded variation, and if $n \theta_{n} \rightarrow \alpha$ as $\theta_{n} \rightarrow 0$, then

$$
s_{n}\left(\theta_{n}\right) \rightarrow(2 / \pi) f(+0) \int_{0}^{\alpha} \frac{\sin t}{t} d t \equiv(2 / \pi) f(+0) I(\alpha) .
$$

In particular, choosing $\alpha$ so that $I(\alpha)=\pi / 2=\int_{0}^{\infty} t^{-1} \sin t d t$ (thus $\int_{\alpha}^{\infty} t^{-1} \sin t d t$ $=0$ ), we get $s_{n}\left(\theta_{n}\right) \rightarrow f(+0)$, which is half of the jump of $f(\theta)$ at $\theta=0$.

On the other hand for $\alpha=\pi$, which gives $I(\alpha)$ its maximal value

$$
s_{n}\left(\theta_{n}\right) \rightarrow(2 / \pi) f(+0) \int_{0}^{\pi} \frac{\sin t}{t} d t=f(+0) \times 1.08949 \ldots .
$$

Thus the limit points of the partial sums as $\theta_{n} \rightarrow 0$ cover an interval which extends beyond $f(+0)$, if $f(+0) \neq 0$. This is called Gibbs' phenomenon.

It was also proved by Fejér and Csillag (for references and further results see Szász [4]) that for functions of bounded variation

$$
n^{-1} \sum_{1}^{n} \nu b_{\nu} \rightarrow(2 / \pi) f(+0), \quad \text { as } n \rightarrow \infty .
$$

These facts suggest the consideration of

$$
s_{n}\left(\theta_{n}\right)=\sum_{1}^{n} \nu b_{\nu} \frac{\sin \nu \theta_{n}}{\nu}, \quad \theta_{n} \rightarrow 0,
$$

as a transform of the sequence $\left\{n b_{n}\right\}$, that is, as a special case of the triangular type transform

Presented to the Society, September 8, 1942; received by the editors September 8, 1942.

(1) Numbers in brackets refer to the literature at the end of this paper. 


$$
T_{n}=\sum_{1}^{n} a_{n \nu} \tau_{\nu},
$$

where now $\tau_{\nu}=\nu b_{\nu}, a_{n \nu}=\nu^{-1} \sin \nu \theta_{n}$. We shall not restrict ourselves to regularity conditions, and we shall not assume convergence of the sequence $\left\{\tau_{n}\right\}$, but merely Cesàro summability of some order. We then seek simple necessary and sufficient conditions for the convergence of the transform $T_{n}$ (in general to a different limit). The application to Fourier sine series yields a generalized Gibbs' phenomenon, and also a new device to determine the generalized jump of a function. Our results are in close relationship with some results of Rogosinski [1, 2].

We consider more generally the transform

$$
T\left(\rho_{n}, \theta_{n}\right)=\sum_{1}^{n} \tau_{\nu \rho_{n}} \nu^{-1} \sin \nu \theta_{n}, \quad \rho_{n} \rightarrow 1, \theta_{n} \rightarrow 0,
$$

which in the case $\tau_{\nu}=\nu b_{\nu}$ becomes $\sum_{1}^{n} \rho_{n}^{\nu} b_{\nu} \sin \nu \theta_{n}=s_{n}\left(\rho_{n}, \theta_{n}\right)$, where $s_{n}(\rho, \theta)$ is the $n$th partial sum of the harmonic series $\sum_{1}^{\infty} \rho^{\nu} b_{\nu} \sin \nu \theta$.

2. Permanency with respect to convergent sequences. It is well known that the convergence of the sequence $\left\{\tau_{n}\right\}$ implies the convergence of the transform $T_{n}$, if and only if

$$
\begin{array}{rlrl}
\lim _{n \rightarrow \infty} a_{n \nu} & =0, & \text { for } \nu=1,2,3, \cdots ; \\
\sum_{\nu=1}^{n}\left|a_{n \nu}\right|=O(1), & \text { as } n \rightarrow \infty ; \\
\lim _{n \rightarrow \infty} \sum_{1}^{n} a_{n \nu}=\sigma \text { exists. } &
\end{array}
$$

We then have $\lim T_{n}=\sigma \lim \tau_{n}$. If we restrict ourselves to sequences $\tau_{n} \rightarrow 0$, then the last condition can be omitted. Applied to (1.6) this yields the necessary and sufficient conditions:

$$
\begin{aligned}
& \sum_{1}^{n} \rho_{n}^{\nu} \nu^{-1}\left|\sin \nu \theta_{n}\right|=O(1), \\
& \lim \sum_{1}^{n} \rho_{n}^{\nu} \nu^{-1} \sin \nu \theta_{n}=\sigma .
\end{aligned}
$$$$
\text { as } n \rightarrow \infty \text {; }
$$

In particular the last condition is $s_{n}\left(\rho_{n}, \theta_{n}\right) \rightarrow \sigma$ for the harmonic series $\sum_{1}^{\infty} \rho^{\nu} \nu^{-1} \sin \nu \theta=\arctan \{(\rho \sin \theta) /(1-\rho \cos \theta)\}$.

We first assume

$$
0<\lim \inf \rho_{n}^{n} \leqq \lim \sup \rho_{n}^{n}<\infty ;
$$


in this case for some $c_{1}>0, c_{2}>0$

$$
c_{1} \sum_{1}^{n} \nu^{-1}|\sin \nu \theta|<\sum_{1}^{n} \rho_{n}^{\nu} \nu^{-1}|\sin \nu \theta|<c_{2} \sum_{1}^{n} \nu^{-1}|\sin \nu \theta|,
$$

thus (2.1) reduces to

$$
\sum_{1}^{n} \nu^{-1}\left|\sin \nu \theta_{n}\right|=O(1)
$$

Now for any $\theta>0$

$$
\sum_{1}^{n} \nu^{-1}|\sin \nu \theta|<n \theta,
$$

hence $n \theta_{n}=O(1)$ implies (2.4). To prove the converse let $\theta_{n}<1<\theta_{n}(n-1)$, and put $\left[\theta_{n}^{-1}\right]=\kappa_{n}=\kappa$, so that $\kappa \leqq \theta_{n}^{-1}<\kappa+1 \leqq n$. Now $\sum_{1}^{n} \nu^{-1}\left|\sin \nu \theta_{n}\right|$ $>(1 / 2) \sum_{1}^{n} \nu^{-1}\left(1-\cos 2 \nu \theta_{n}\right)$, and

$$
\begin{aligned}
\left|\sum_{1}^{n} \nu^{-1} \cos 2 \nu \theta_{n}\right| & <\sum_{1}^{k} \nu^{-1}+\left|\sum_{\kappa+1}^{n} \nu^{-1} \cos 2 \nu \theta_{n}\right| \\
& <1+\log \kappa+(1 /(\kappa+1)) \max _{\kappa<\lambda \leqq n}\left|\sum_{\kappa+1}^{\lambda} \cos 2 \nu \theta_{n}\right| \\
& <1+\log \theta_{n}^{-1}+\theta_{n} / \sin \theta_{n}<3-\log \theta_{n} .
\end{aligned}
$$

Thus

$$
2 \sum_{1}^{n} \nu^{-1}\left|\sin \nu \theta_{n}\right|>\log n+\log \theta_{n}-3=-3+\log \left(n \theta_{n}\right) ;
$$

hence (2.4) implies $n \theta_{n}=O(1)$. For null sequences only this is required.

To satisfy (2.2) consider the case that 0 is a limit point of the sequence $\left\{n \theta_{n}\right\}$; for a subsequence of indices $n: n \theta_{n} \rightarrow 0$, and for that subsequence, using (2.5)

$$
\sum_{1}^{n} \rho_{n} \nu^{-1} \sin \nu \theta_{n}=O\left(\sum_{1}^{n} \nu^{-1}\left|\sin \nu \theta_{n}\right|\right)=O\left(n \theta_{n}\right)=o(1) \text {. }
$$

Hence $\sigma$, if it exists, is 0 and then every convergent sequence is transformed into a null sequence. Next assume $\lim \inf n \theta_{n}>0$. We choose a subsequence of integers $n=n^{\prime}$ for which $\rho_{n}^{n}$ and $n \theta_{n}$ have limits $n^{\prime} \theta_{n^{\prime}} \rightarrow \beta>0, \rho_{n^{\prime}}^{n^{\prime}} \rightarrow e^{\gamma}$ say; by (2.3) $\gamma$ is finite. Furthermore from $\log \rho /(\rho-1) \rightarrow 1$ as $\rho \rightarrow 1, n^{\prime}\left(\rho_{n^{\prime}}-1\right) \rightarrow \gamma$.

Suppose first $\gamma=0$, that is $\rho_{n^{\prime}}^{n^{\prime}} \rightarrow 1$, and $n^{\prime}\left(\rho_{n^{\prime}}-1\right) \rightarrow 0$. Now, as $n$ runs through the sequence $\left\{n^{\prime}\right\}$

$$
\left|\sum_{1}^{n}\left(\rho_{n}^{\prime}-1\right) \nu^{-1} \sin \nu \theta_{n}\right|<\left|\rho_{n}-1\right| O(n) \sum_{1}^{n} \nu^{-1}\left|\sin \nu \theta_{n}\right|=o(1) O\left(n \theta_{n}\right)=o(1),
$$


hence

$$
\lim \sum_{1}^{n} \rho_{n}^{\prime} \nu^{-1} \sin \nu \theta_{n}=\lim \sum_{1}^{n} \nu^{-1} \sin \nu \theta_{n}
$$

for $n=n^{\prime} \rightarrow \infty$, if either side exists. But

$$
\begin{aligned}
\sum_{1}^{n} \nu^{-1} \sin \nu \theta & =\int_{0}^{0}\left(\sum_{1}^{n} \cos \nu t\right) d t=-(1 / 2) \theta+\int_{0}^{0} \frac{\sin (n+1 / 2) t}{2 \sin (t / 2)} d t \\
& =-(1 / 2) \theta+\int_{0}^{(n+1 / 2) 0} \frac{\sin u d u}{(2 n+1) \sin (u /(2 n+1))},
\end{aligned}
$$

hence

$$
\begin{aligned}
\sum_{1}^{n} \nu^{-1} \sin \nu \theta_{n} & =-(1 / 2) \theta_{n}+\int_{0}^{\beta} \frac{\sin u}{u} \cdot \frac{u d u}{(2 n+1) \sin (u /(2 n+1))}+o(1) \\
& \rightarrow \int_{0}^{\beta} \frac{\sin u}{u} d u
\end{aligned}
$$

as $n \rightarrow \infty$ through the sequence $\left\{n^{\prime}\right\}$. The consideration of the case $\gamma \neq 0$ remains; we write

$$
\begin{aligned}
\sum_{1}^{n} \rho^{\prime} \nu^{-1} \sin \nu \theta \\
\quad=\int_{0}^{0}\left(\sum_{1}^{n} \rho^{\prime} \cos \nu t\right) d t \\
\quad=\int_{0}^{0} \frac{\cos t-\rho^{2}+\rho^{n+2} \cos n t-\rho^{n+1} \cos (n+1) t}{1-2 \rho \cos t+\rho^{2}} d t \\
\quad=\int_{0}^{0} \frac{1-\rho^{2}-(1-\cos t)+\rho^{n+1}[\cos n t-\cos (n+1) t]-(1-\rho) \rho^{n+1} \cos n t}{(1-\rho)^{2}+2 \rho(1-\cos t)} d t,
\end{aligned}
$$

thus

$$
\begin{aligned}
\sum_{1}^{n} \rho^{n} \nu^{-1} \sin \nu \theta= & \left(1-\rho^{2}\right) \int_{0}^{0} \frac{d t}{(1-\rho)^{2}+4 \rho \sin ^{2}(t / 2)} \\
& -2 \int_{0}^{0} \frac{\sin ^{2}(t / 2) d t}{(1-\rho)^{2}+4 \rho \sin ^{2}(t / 2)} \\
& -(1-\rho) \rho^{n+1} \int_{0}^{\theta} \frac{\cos n t d t}{(1-\rho)^{2}+4 \rho \sin ^{2}(t / 2)} \\
& +2 \rho^{n+1} \int_{0}^{0} \frac{\sin (t / 2) \sin (n+1 / 2) t d t}{(1-\rho)^{2}+4 \rho \sin ^{2}(t / 2)} .
\end{aligned}
$$


Now

$$
\int_{0}^{\theta_{n}} \frac{\sin ^{2}(t / 2) d t}{\left(1-\rho_{n}\right)^{2}+4 \rho_{n} \sin ^{2}(t / 2)}<\frac{\theta_{n}}{4 \rho_{n}} \rightarrow 0 \quad \text { as } n \rightarrow \infty \text {. }
$$

Next

$$
\begin{aligned}
& \left(\rho_{n}^{2}-1\right) \int_{0}^{\theta_{n}} \frac{d t}{\left(1-\rho_{n}\right)^{2}+4 \rho_{n} \sin ^{2}(t / 2)} \\
& \quad=n\left(\rho_{n}-1\right)\left(\rho_{n}+1\right) \int_{0}^{n \theta_{n}} \frac{d u}{n^{2}\left[\left(\rho_{n}-1\right)^{2}+4 \rho_{n} \sin ^{2}(u / 2 n)\right]} \\
& \quad \rightarrow 2 \gamma \int_{0}^{\beta} \frac{d u}{\gamma^{2}+u^{2}}=2 \arctan (\beta / \gamma) .
\end{aligned}
$$

Similarly

$$
\begin{aligned}
& \left(\rho_{n}-1\right) \rho_{n}^{n+1} \int_{0}^{\theta_{n}} \frac{\cos n t d t}{\left(1-\rho_{n}\right)^{2}+4 \rho_{n} \sin ^{2}(t / 2)} \\
& \quad=n\left(\rho_{n}-1\right) \rho_{n}^{n+1} \int_{0}^{n \theta_{n}} \frac{\cos u d u}{n^{2}\left[\left(\rho_{n}-1\right)^{2}+4 \rho_{n} \sin ^{2}(u / 2 n)\right]} \rightarrow \gamma e^{\gamma} \int_{0}^{\beta} \frac{\cos u d u}{\gamma^{2}+u^{2}},
\end{aligned}
$$

and

$\rho_{n}^{n+1} \int_{0}^{\theta_{n}} \frac{\sin (t / 2) \sin (n+1 / 2) t d t}{\left(1-\rho_{n}\right)^{2}+4 \rho_{n} \sin ^{2}(t / 2)}$

$$
\begin{aligned}
= & \rho_{n}^{n+1} \int_{0}^{(n+1 / 2) \theta_{n}} \frac{(2 n+1) \sin \{u /(2 n+1)\} \cdot \sin u d u}{n(2 n+1)\left[\left(\rho_{n}-1\right)^{2}+4 \rho_{n} \sin ^{2}\{u /(2 n+1)\}\right]} \\
& \rightarrow(1 / 2) e^{\gamma} \int_{0}^{\beta} \frac{u \sin u d u}{\gamma^{2}+u^{2}} .
\end{aligned}
$$

Summarizing

$$
\begin{aligned}
\sum_{1}^{n} \rho_{n}^{\nu} \nu^{-1} \sin \nu \theta_{n} & \rightarrow \int_{0}^{\beta} \frac{\gamma e^{\gamma} \cos u+e^{\gamma} u \sin u-2 \gamma}{\gamma^{2}+u^{2}} d u \\
& =\int_{0}^{\beta / \gamma} \frac{e^{\gamma}(t \sin \gamma t+\cos \gamma t)-2}{1+t^{2}} d t .
\end{aligned}
$$

The case (2.3) is now completely discussed. We next assume

$$
\limsup _{n \rightarrow \infty} \rho_{n}^{n}=\infty,
$$

so that for a subsequence $n=n^{\prime}: \rho_{n^{\prime}}^{n^{\prime}} \rightarrow \infty$. We first prove that (2.1) implies $n^{\prime} \theta_{n^{\prime}} \rightarrow 0$. Otherwise for a subsequence $n^{\prime \prime}$ of $n^{\prime}: n^{\prime \prime} \theta_{n^{\prime \prime}} \rightarrow \beta>0$. For these indices 


$$
\sum_{1}^{n} \rho_{n}^{\nu} \nu^{-1}\left|\sin \nu \theta_{n}\right|>\sum_{\nu \leqq \alpha / \theta_{n}} \rho_{n}^{\nu} \nu^{-1}\left|\sin \nu \theta_{n}\right|,
$$

where $\alpha$ is so chosen that $0<\alpha<\beta$ and $\alpha \leqq \pi / 2$. Now

$$
\sum_{\nu \leq \alpha / \theta_{n}} \rho_{n}^{\prime} \nu^{-1}\left|\sin \nu \theta_{n}\right|>(2 / \pi) \theta_{n} \sum \rho_{n}^{\prime}=\frac{2 \rho_{n} \theta_{n}}{\pi} \frac{\rho_{n}^{\left[\alpha \theta_{n}^{-1}\right]}-1}{\rho_{n}-1},
$$

hence (2.1) implies

$$
\theta_{n} \rho_{n}^{\alpha n / 2 \beta}=O\left(\rho_{n}-1\right)
$$

which by virtue of $\log \rho_{n} /\left(\rho_{n}-1\right) \rightarrow 1$ yields $n \theta_{n}=o(1)$. Furthermore

$$
(2 / \pi) \theta_{n} \sum \rho_{n}^{\nu}<\sum_{1}^{n} \rho_{n}^{\nu} \nu^{-1}\left|\sin \nu \theta_{n}\right|<\theta_{n} \sum_{1}^{n} \rho_{n}^{\nu}
$$

thus, if for a subsequence of indices $\rho_{n}^{n} \rightarrow \infty$, then for these indices (2.1) is equivalent to

$$
\theta_{n} \rho_{n}^{n}=O\left(\rho_{n}-1\right)
$$

If this condition is satisfied, then in view of $n \theta_{n} \rightarrow 0$

$$
\begin{aligned}
0 & <\theta_{n} \sum_{1}^{n} \rho_{n}^{\nu}-\sum_{1}^{n} \rho_{n}^{\nu} \nu^{-1} \sin \nu \theta_{n}=\theta_{n} \sum_{1}^{n} \rho_{n}^{\nu}\left(1-\frac{\sin \nu \theta_{n}}{\nu \theta_{n}}\right) \\
& <\theta_{n}\left(1-\frac{\sin n \theta_{n}}{n \theta_{n}}\right) \sum_{1}^{n} \rho_{n}^{\nu} \rightarrow 0,
\end{aligned}
$$

hence (2.2) holds if and only if $\lim \theta_{n} \rho_{n}^{n} /\left(\rho_{n}-1\right)$ exists, which is then the value of $\sigma$.

Finally assume that $\lim$ inf $\rho_{n}^{n}=0$; thus for a subsequence of indices $\rho_{n}^{n} \rightarrow 0$ $(\gamma=-\infty)$. If $n \theta_{n}=O(1)$, then $\sum_{1}^{n} \rho_{n}^{\nu} \nu^{-1}\left|\sin \nu \theta_{n}\right|=O\left(\sum_{1}^{n} \nu^{-1}\left|\sin \nu \theta_{n}\right|\right)=O(1)$, which is (2.1). If on the other hand for a subsequence $n \theta_{n} \rightarrow \infty$, then

$$
\sum_{1}^{n} \rho_{n}^{\prime} \nu^{-1}\left|\sin \nu \theta_{n}\right|>\sum_{\theta_{n}^{-1}<\nu}^{\leqq n} \rho_{n}^{\nu} \nu^{-1} \frac{1-\cos 2 \nu \theta_{n}}{2} ;
$$

but $\rho_{n}^{\nu} \nu^{-1} \downarrow$ as $\nu \uparrow$, hence for $\theta_{n}<1$

$$
\begin{aligned}
\left|\sum_{\theta_{n}^{-1}<\nu}^{\leqq n} \rho_{n}^{\prime \nu} \cos 2 \nu \theta_{n}\right| & <\rho_{n}^{1+\left[\theta_{n}^{-1}\right]} \frac{1}{1+\left[\theta_{n}^{-1}\right]} \max _{\lambda \geqq_{n}}\left|\sum_{\theta_{n}^{-1}<\nu}^{\leqq \lambda} \cos 2 \nu \theta_{n}\right| \\
& <\frac{\theta_{n}}{\sin \theta_{n}}<\pi / 2 .
\end{aligned}
$$

Furthermore 


$$
\begin{aligned}
\sum_{\theta_{n}^{-1}<\nu}^{\leq n} \rho_{n}^{\prime} \nu^{-1} & =\sum_{\theta_{n}^{-1}<\nu}^{\leq n} \nu^{-1} \exp \left(\nu \log \rho_{n}\right)>\sum \int_{\nu}^{\nu+1} u^{-1} \exp \left(-u \log \rho_{n}^{-1}\right) d u \\
& =\int_{1+\left[\theta_{n}^{-1}\right]}^{n+1} u^{-1} \exp \left(-u \log \rho_{n}^{-1}\right) d u=\int_{\left(1+\left[\theta_{n}^{-1}\right]\right) \log \rho_{n}^{-1}}^{(n+1) \log \rho_{n}^{-1}} t^{-1} e^{-t} d t ;
\end{aligned}
$$

thus in this case $(2.1)$ implies $\theta_{n}=O\left(\log 1 / \rho_{n}\right)$, or $\theta_{n}=O\left(1-\rho_{n}\right)$. If this condition is satisfied, then

$$
\sum_{1}^{n} \rho_{n}^{\nu} \nu^{-1}\left|\sin \nu \theta_{n}\right|=O\left(\theta_{n} \sum_{1}^{n} \rho_{n}^{\nu}\right)=O\left(\theta_{n} /\left(1-\rho_{n}\right)\right)=O(1)
$$

hence (2.1) holds. To satisfy. (2.2) now, we note that

$$
\sum_{n+1}^{\infty} \rho_{n}^{n} \nu^{-1} \sin \nu \theta_{n}=O\left(\theta_{n} \frac{\rho_{n}^{n}}{1-\rho_{n}}\right)=O\left(\rho_{n}^{n}\right)=o(1),
$$

hence (2.2) holds if and only if

$$
\sum_{1}^{\infty} \rho_{n}^{\nu} \nu^{-1} \sin \nu \theta_{n}=\arctan \frac{\rho_{n} \sin \theta_{n}}{1-\rho_{n} \cos \theta_{n}}
$$

has a limit, and $\sigma$ is then this limit. But

$$
\frac{\rho_{n} \sin \theta_{n}}{1-\rho_{n} \cos \theta_{n}}=\frac{\rho_{n} \sin \theta_{n}}{1-\rho_{n}+\rho_{n}\left(1-\cos \theta_{n}\right)} \sim \frac{\theta_{n}}{1-\rho_{n}} \cdot \frac{1}{1+O\left(1-\rho_{n}\right)} \sim \frac{\theta_{n}}{1-\rho_{n}},
$$

hence $\sigma$ exists, if and only if $\lim \theta_{n} /\left(1-\rho_{n}\right)=\delta<+\infty$. We then have $\sigma=\lim \arctan \left\{\theta_{n} /\left(1-\rho_{n}\right)\right\}=\arctan \delta$. To summarize our results put

(a)

$$
\sigma(\beta, 0)=\int_{0}^{\beta} \frac{\sin u}{u} d u,
$$

$$
\sigma(\beta, \gamma)=\int_{0}^{\beta} \frac{\gamma e^{\gamma} \cos u+e^{\gamma} \sin u-2 \gamma}{\gamma^{2}+u^{2}} d u, \text { for finite } \gamma \neq 0
$$

(b)

$$
\sigma(0, \infty)=\lim \frac{\theta_{n} \rho_{n}^{n}}{\rho_{n}-1}
$$

(c) $\quad \sigma(\delta,-\infty)=\lim \arctan \frac{\theta_{n}}{1-\rho_{n}}=\arctan \delta<\pi / 2$.

We then have

THEOREM 1. Necessary and sufficient conditions that for every convergent sequence $n b_{n} \rightarrow \tau$ the transform $\sum_{1}^{n} \rho_{n}^{v} b_{v} \sin \nu \theta_{n}$ has a limit, are that one of the following three cases holds: 

(a') $n\left(\rho_{n}-1\right) \rightarrow \gamma$ finite, $n \theta_{n} \rightarrow \beta<\infty$,
(b') $n\left(\rho_{n}-1\right) \rightarrow+\infty, \lim \theta_{n} \rho_{n}^{n}\left(\rho_{n}-1\right)^{-1}$ exists,
(c') $n\left(\rho_{n}-1\right) \rightarrow-\infty, \lim \theta_{n}\left(1-\rho_{n}\right)^{-1}=\delta$ exists, $0 \leqq \delta<\infty$.

The limit of the transform is then $\tau \sigma$, where $\sigma$ is defined above for the respective cases. Different subsequences may belong to different cases $(\beta, \gamma)$ if only the corresponding $\sigma$ attain the same value, and with the restriction $n \theta_{n}=O(1)$ in case $\left(\mathrm{a}^{\prime}\right)$.

3. Permanency with respect to $(C, \kappa)$ summability. Given the sequence $\left\{\tau_{n}\right\}$, write

also

$$
\tau_{n}^{0}=\tau_{n}, \quad \tau_{n}^{k}=\sum_{v=1}^{n} \tau_{n}^{k-1}, \quad n, \kappa=1,2,3, \cdots ;
$$

$$
A_{n}^{\kappa}=C_{n+\kappa, n}=\frac{(\kappa+1) \cdots(\kappa+n)}{n !} \sim \frac{n^{\kappa}}{\kappa !}, \quad \text { as } n \rightarrow \infty .
$$

The sequence $\left\{\tau_{n}\right\}$ is summable $(C, \kappa)$ to the value $\tau$, if $\tau_{n}^{\kappa} / A_{n}^{\kappa} \rightarrow \tau$ as $n \rightarrow \infty$; $(C, 0)$ is evidently convergence.

We write

$$
\Delta^{0} \tau_{n}=\tau_{n}, \quad \Delta^{1} \tau_{n}=\Delta \tau_{n}=\tau_{n}-\tau_{n+1}, \quad \Delta^{*} \tau_{n}=\Delta\left(\Delta^{\kappa-1} \tau_{n}\right) ;
$$

then by induction

$$
\Delta^{*} \tau_{n}=\sum_{\nu=0}^{k}(-1)^{\nu} C_{k, \nu} \tau_{n+\nu}, \quad \quad \kappa=0,1,2, \ldots .
$$

Abel's transformation yields for finite sums

$$
\sum_{1}^{n} \alpha_{\nu} \tau_{\nu}=\sum_{1}^{n} \tau_{\nu}^{1} \Delta \alpha_{\nu}=\sum_{1}^{n} \tau_{\nu}^{2} \Delta^{2} \alpha_{\nu}=\cdots,
$$

where $\alpha_{n+1}=0, \alpha_{n+2}=0, \cdots$. Applying this to (1.5) we get

$$
T_{n}=\sum_{n=1}^{n} \tau_{v}^{*} \Delta^{*} a_{n v}
$$

where $a_{n \nu}=0$ for $\nu>n$. Thus the transform converges for every $(C, \kappa)$ summable sequence if in addition to the conditions of $\$ 2$

$$
\sum_{n=1}^{n} A_{\nu}^{*}\left|\Delta^{k} a_{n v}\right|=O(1) \quad \text { as } n \rightarrow \infty .
$$

In particular for the transform (1.6) we have the conditions (2.1), (2.2) and

$$
\sum_{n=1}^{n-k} A_{\nu}^{\alpha}\left|\Delta^{*} \rho_{n}^{\nu} \nu^{-1} \sin \nu \theta_{n}\right|+\sum_{n-\alpha+1}^{n} A_{\nu}^{*}\left|\delta_{\nu}\right|=O(1) \quad \text { as } n \rightarrow \infty,
$$


where, from (3.2)

$$
\delta_{\lambda}=\sum_{\nu=0}^{n-\lambda}(-1)^{\nu} C_{\kappa, \nu} \rho_{n}^{\lambda+\nu} \frac{\sin (\lambda+\nu) \theta_{n}}{\lambda+\nu}, \quad n-\kappa<\lambda \leqq n .
$$

We first consider $(C, 1)$ summability $(\kappa=1)$. Now (3.3) becomes

or

$$
\sum_{1}^{n-1}(\nu+1)\left|\Delta \rho_{n}^{\prime} \nu^{-1} \sin \nu \theta_{n}\right|+(n+1) \rho_{n}^{n} n^{-1}\left|\sin n \theta_{n}\right|=O(1),
$$

(3.4) $\sum_{1}^{n-1} \nu\left|\rho_{n}^{\nu} \nu^{-1} \sin \nu \theta_{n}-\rho_{n}^{\nu+1}(\nu+1)^{-1} \sin (\nu+1) \theta_{n}\right|+\rho_{n}^{n}\left|\sin n \theta_{n}\right|=O(1)$.

We consider in succession the different cases of Theorem 1.

$\left(\mathrm{a}^{\prime}\right)$ For a sequence of indices $n \theta_{n} \rightarrow \beta<\infty, n\left(\rho_{n}-1\right) \rightarrow \gamma$ finite, that is, $\rho_{n}^{n} \rightarrow e^{r}>0$. Thus $\rho_{n}^{n} \sin n \theta_{n}$ is $O(1)$, and

$$
\begin{aligned}
\sum_{1}^{n-1} \nu \rho_{n}^{\prime} \mid \nu^{-1} \sin \nu \theta_{n} & -\rho_{n}(\nu+1)^{-1} \sin (\nu+1) \theta_{n} \mid \\
\leqq & \sum_{1}^{n-1} \nu \rho_{n}^{\nu}\left|\nu^{-1} \sin \nu \theta_{n}-(\nu+1)^{-1} \sin (\nu+1) \theta_{n}\right| \\
& +\left|1-\rho_{n}\right| \sum_{1}^{n-1} \rho_{n}^{\nu}\left|\sin (\nu+1) \theta_{n}\right| .
\end{aligned}
$$

Now

$$
\left|1-\rho_{n}\right| \sum_{1}^{n-1} \rho_{n}^{\prime}\left|\sin (\nu+1) \theta_{n}\right|<\left|1-\rho_{n}\right| \sum_{1}^{n} \rho_{n}^{\prime}<\rho_{n}\left|1-\rho_{n}^{n}\right|=O(1),
$$

and

$$
\begin{aligned}
& \nu\left|\nu^{-1} \sin \nu \theta_{n}-(\nu+1)^{-1} \sin (\nu+1) \theta_{n}\right| \\
& \quad=\left|(\nu+1)^{-1} \sin (\nu+1) \theta_{n}-2 \sin (1 / 2) \theta_{n} \cos ((2 \nu+1) / 2) \theta_{n}\right|<2 \theta_{n}
\end{aligned}
$$

hence

$$
\sum_{1}^{n-1} \nu \rho_{n}^{\nu}\left|\nu^{-1} \sin \nu \theta_{n}-(\nu+1)^{-1} \sin (\nu+1) \theta_{n}\right|<2 \theta_{n} \sum_{1}^{n} \rho_{n}^{\nu}=O\left(n \theta_{n}\right)=O(1) .
$$

Hence in this case no additional condition results.

(b') $n\left(\rho_{n}-1\right) \rightarrow+\infty, \theta_{n} \rho_{n}^{n}\left(\rho_{n}-1\right)^{-1} \rightarrow \sigma$. Hence $n \theta_{n} \rightarrow 0$, and now $\rho_{n}^{n} \sin n \theta_{n}$ $=O(1)$ is equivalent to $n \theta_{n} \rho_{n}^{n}=O(1)$. Thus $\theta_{n} \rho_{n}^{n}\left(\rho_{n}-1\right)^{-1}=n \theta_{n} \rho_{n}^{n} n^{-1}\left(\rho_{n}-1\right)^{-1}$ $\rightarrow 0$, that is, $\sigma=0$. Now

$$
\begin{aligned}
\left|1-\rho_{n}\right| \sum_{1}^{n-1} \rho_{n}^{\prime}\left|\sin (\nu+1) \theta_{n}\right| & =O\left[\left(\rho_{n}-1\right) \theta_{n} \sum_{1}^{n} \nu \rho_{n}^{\prime}\right] \\
& =O\left[\theta_{n} \rho_{n}^{n}\left(\rho_{n}-1\right)^{-1}\right]=o(1)
\end{aligned}
$$


furthermore

$$
\theta_{n} \sum_{1}^{n} \rho_{n}^{\prime}=O\left[\theta_{n} \rho_{n}^{n}\left(\rho_{n}-1\right)^{-1}\right]=o(1)
$$

hence (3.4) holds. Finally:

(c') If $\lim \theta_{n} /\left(1-\rho_{n}\right)<\infty$ exists, and $n\left(\rho_{n}-1\right) \rightarrow-\infty$, that is, $\rho_{n}^{n} \rightarrow 0$, then $\rho<\rho_{n}^{n} \sin n \theta_{n} \rightarrow 0,\left|1-\rho_{n}\right| \sum_{1}^{n-1} \rho_{n}^{\nu}\left|\sin (\nu+1) \theta_{n}\right|<\left(1-\rho_{n}\right) \sum_{0}^{\infty} \rho_{n}^{\nu}=1$, and

$$
\theta_{n} \sum_{1}^{n} \rho_{n}^{\nu}<\frac{\theta_{n}}{1-\rho_{n}}=O(1)
$$

No additional condition appears in this case. Summarizing, we have

THEOREM 2. Necessary and sufficient conditions that when $\lim n^{-1} \sum_{1}^{n} \nu b_{\nu}=\tau$ exists the transform $\sum_{1}^{n} \rho_{n}^{\nu} b_{\nu} \sin \nu \theta_{n}$ has a limit: $\tau \sigma$, are either of the alternatives:

$\left(\mathrm{a}^{\prime \prime}\right) n\left(\rho_{n}-1\right) \rightarrow \gamma$, finite, $n \theta_{n} \rightarrow \beta<\infty$,

$\left(\mathrm{b}^{\prime \prime}\right) n\left(\rho_{n}-1\right) \rightarrow+\infty, n \theta_{n} \rho_{n}^{n}=O(1)$,

(c' $n\left(\rho_{n}-1\right) \rightarrow-\infty, \lim \theta_{n}\left(1-\rho_{n}\right)^{-1}$ exists.

The value of $\sigma$ is in the cases $\left(a^{\prime \prime}\right)$ and $\left(c^{\prime \prime}\right)$ given by (a) and (c). In case $\left(b^{\prime \prime}\right) \sigma=0$. Different subsequences may belong to different cases if only $\sigma$ has the same value, with the restriction $n \theta_{n}=O(1)$ in case $\left(\mathrm{a}^{\prime \prime}\right)$.

We now consider $(C, \kappa)$ summability for $\kappa>1$. First of all, to satisfy (3.3) we must have

$$
n^{\kappa} \sum_{\nu=0}^{m}(-1)^{\nu} C_{\kappa, \nu} \rho_{n}^{n-m+\nu} \frac{\sin (n-m+\nu) \theta_{n}}{n-m+\nu}=O(1), m=0,1, \cdots, \kappa-1
$$

Or

$$
\begin{array}{r}
n^{k-1} \rho_{n}^{n} \sin n \theta_{n}=O(1), \\
n^{k} \rho_{n}^{n}\left\{\frac{\sin (n-1) \theta_{n}}{n-1}-\kappa \rho_{n} \frac{\sin n \theta_{n}}{n}\right\}=O(1), \\
n^{k} \rho_{n}^{n}\left\{\frac{\sin (n-\kappa+1) \theta_{n}}{n-\kappa+1}-\ldots+(-\ldots)^{\kappa-1} \kappa_{n}^{k-1} \frac{\sin n \theta_{n}}{n}\right\}=O(1) .
\end{array}
$$

This is equivalent to

$$
\begin{aligned}
n^{k-1} \rho_{n}^{n} \sin n \theta_{n} & =O(1), \\
n^{k-1} \rho_{n}^{n} \sin (n-1) \theta_{n} & =O(1), \\
\cdot . \cdot . \cdot . \cdot . \cdot . \cdot . \cdot & \cdot . \cdot \\
n^{k-1} \rho_{n}^{n} \sin (n-\kappa+1) \theta_{n} & =O(1) .
\end{aligned}
$$


In case $\left(a^{\prime \prime}\right)$ the first condition becomes $\sin n \theta_{n}=O\left(n^{1-x}\right)$, as $n \rightarrow \infty$; in particular $\sin n \theta_{n} \rightarrow 0$, thus in view of $\left(\mathrm{a}^{\prime \prime}\right) n \theta_{n} \rightarrow \lambda \pi, \lambda$ a positive integer or zero. On putting $n \theta_{n}=\lambda \pi+\epsilon_{n}$, we get $\sin \epsilon_{n}=O\left(n^{1-k}\right)$, or $n \theta_{n}-\lambda \pi=O\left(n^{1-k}\right)$. From the second condition now $\cos n \theta_{n} \sin \theta_{n}=O\left(n^{1-\alpha}\right)$, as $n \rightarrow \infty$, or $\lambda \pi+\epsilon_{n}=O\left(n^{2-\alpha}\right)$; hence for $k=2,(3.6)$ reduces to

$$
n \theta_{n}=\lambda \pi+O\left(n^{-1}\right)
$$

For $\kappa>2$ we must have

$$
n \theta_{n}-\lambda \pi=\epsilon_{n}=O\left(n^{1-x}\right) \text { and } \lambda \pi+\epsilon_{n}=O\left(n^{2-x}\right),
$$

hence $\lambda=0$, and

$$
n \theta_{n}=O\left(n^{1-x}\right)
$$

It then follows that

$$
n^{k-1} \sin (n-\nu) \theta_{n}=O(1) \quad \text { for } \nu=0,1, \cdots, k-1 .
$$

Furthermore, for the rest of (3.3)

$$
\sum_{1}^{n-\alpha} A_{\nu}^{\alpha}\left|\Delta^{\alpha} \rho_{n}^{\prime} \nu^{-1} \sin \nu \theta_{n}\right|=O\left(\sum_{1}^{n-\alpha} \nu^{\alpha}\left|\Delta^{\alpha} \rho_{n}^{n} \nu^{-1} \sin \nu \theta_{n}\right|\right) \text {. }
$$

Now

$$
\Delta^{*} \rho^{\nu} \nu^{-1} \sin \nu \theta=\Delta^{*} \rho^{\nu} \int_{0}^{\theta} \cos \nu t d t=R \int_{0}^{\theta} \Delta^{\alpha} z^{\nu} d t, \quad z=\rho e^{i t},
$$

and, using (3.2)

$$
\Delta^{\alpha} \rho^{y} \nu^{-1} \sin \nu \theta=R \int_{0}^{\theta} \sum_{\lambda=0}^{k}(-1)^{\lambda} C_{k, \lambda} z^{\nu+\lambda} d t=R \int_{0}^{\theta} z^{\nu}(1-z)^{\alpha} d t,
$$

hence

Thus

$$
\begin{aligned}
\left|\Delta^{\alpha} \rho^{\nu} \nu^{-1} \sin \nu \theta\right| & <\rho^{\nu} \int_{0}^{\theta}\left|1-\rho e^{i t}\right|^{\alpha} d t<\rho^{\nu} \int_{0}^{\theta}\left\{(1-\rho)^{2}+\rho t^{2}\right\} \times / 2 d t \\
& <\theta \rho^{\nu}\left\{(1-\rho)^{2}+\rho \theta^{2}\right\} \alpha / 2 .
\end{aligned}
$$

and, from $\rho_{n}^{n}=O(1)$,

$$
\begin{aligned}
\sum_{1}^{n-k} \nu^{\alpha}\left|\Delta^{\alpha} \rho_{n}^{\prime} \nu^{-1} \sin \nu \theta_{n}\right| & <\left(\sum_{1}^{n} \nu^{\alpha} \rho_{n}^{\nu}\right)\left\{\left(1-\rho_{n}\right)^{2}+\rho_{n} \theta_{n}^{2}\right\}^{\alpha / 2} \theta_{n} \\
& <\left\{n^{2}\left(1-\rho_{n}\right)^{2}+\rho_{n} n^{2} \theta_{n}^{2}\right\}^{k / 2} \theta_{n} \sum_{1}^{n} \rho_{n}^{\prime} \\
& =O\left(\theta_{n} \sum_{1}^{n} \rho_{n}^{\prime}\right)
\end{aligned}
$$




$$
\theta_{n} \sum_{1}^{n} \rho_{n}^{\prime}=O\left(n \theta_{n}\right)=O(1)
$$

Hence in case $\left(a^{\prime \prime}\right)$ the additional condition is (3.7) for $\kappa=2$, and $\left(3.7^{\prime}\right)$ for $\kappa>2$.

In case $\left(\mathrm{b}^{\prime \prime}\right): n\left(\rho_{n}-1\right) \rightarrow+\infty, n \theta_{n} \rho_{n}^{n}=O(1)$, as $n \rightarrow \infty$; hence $\rho_{n}^{n} \rightarrow+\infty$, and $n \theta_{n} \rightarrow 0$. Now (3.6) becomes

$$
n^{*} \rho_{n}^{n} \theta_{n}=O(1)
$$

For large $n$ evidently $\rho_{n}>1$, and

$$
\theta_{n} \sum_{1}^{n} \dot{\rho}_{n}^{\prime}<n \theta_{n} \rho_{n}^{n}=O(1)
$$

(from (3.9)). In view of (3.8) now (3.3) holds. Thus in this case the additional condition is (3.9) (for $\kappa \geqq 2$ ).

Finally, in case $\left(c^{\prime \prime}\right): n\left(\rho_{n}-1\right) \rightarrow-\infty$ (that is $\left.\rho_{n}^{n} \rightarrow 0\right)$, and $\lim \theta_{n} /\left(1-\rho_{n}\right)$ $=\delta<\infty$ exists. Now $\rho_{n}^{n}<1 /\left(n\left(1-\rho_{n}\right)\right)$, hence $n \theta_{n} \rho_{n}^{n}=O(1)$; thus for $\kappa=2$ condition (3.6) reduces to $n \rho_{n}^{n} \sin n \theta_{n}=O(1)$. While for $\kappa>2$ (3.6) reduces to $n^{k-1} \rho_{n}^{n} \sin n \theta_{n}=O(1)$ and $n^{k-1} \theta_{n} \rho_{n}^{n}=O(1)$. Furthermore, as $\rho_{n}<1, \theta_{n} \sum_{0}^{n} \rho_{n}^{p}$ $<\theta_{n} /\left(1-\rho_{n}\right)=O(1)$, hence, in view of (3.8) now (3.3) is satisfied.

We summarize our results in

THEOREM 3. In order that $\lim \sum_{1}^{n} \rho_{n}^{\nu} b_{\nu} \sin \nu \theta_{n}=\tau \sigma$ exists, whenever $(C, \kappa)$ $\lim n b_{n}=\tau$ for some $\kappa \geqq 2$, necessary and sufficient conditions are the alternatives:

$\left(\mathrm{a}^{\prime \prime \prime}\right) n\left(\rho_{n}-1\right) \rightarrow \gamma$, finite, and for $\kappa=2: n \theta_{n}=\lambda \pi+O\left(n^{-1}\right), \lambda$ an integer, for $\kappa>2: \theta_{n}=O\left(n^{-\kappa}\right)$;

$\left(\mathrm{b}^{\prime \prime \prime}\right) n\left(\rho_{n}-1\right) \rightarrow+\infty, n^{x} \rho_{n}^{n} \theta_{n}=O(1)$;

(c' $\left.c^{\prime \prime \prime}\right) n\left(\rho_{n}-1\right) \rightarrow-\infty, \quad \lim \theta_{n} /\left(1-\rho_{n}\right)=\delta<\infty$ exists, and for $\kappa=2$ : $n \rho_{n}^{n} \sin n \theta_{n}=O(1)$, for $\kappa>2: n^{\kappa-1} \rho_{n}^{n}\left(\theta_{n}+\left|\sin n \theta_{n}\right|\right)=O(1)$.

The value of $\sigma$ is given in case $\left(a^{\prime \prime \prime}\right)$ by $(a)$, where for $\kappa=2: \beta=\lambda \pi$, for $\kappa>2: \beta=0, \sigma=0$; in case $\left(b^{\prime \prime \prime}\right): \sigma=0$; in case $\left(c^{\prime \prime \prime}\right): \sigma=\arctan \delta$.

4. Application to Fourier series. First consider a function of bounded variation and its Fourier sine series (1.1). It follows from the introduction that $\lim n b_{n}$, if it exists, is $(2 / \pi) f(+0)$. Under the assumptions of Theorem 1 on $\rho_{n}$ and $\theta_{n}, s_{n}\left(\rho_{n}, \theta_{n}\right) \rightarrow \tau \sigma=(2 \sigma / \pi) f(+0)$. In particular whenever $\sigma>\pi / 2$, then we have an analogue of Gibbs' phenomenon. It is known that for functions of bounded variation

$$
(1 / n) \sum_{1}^{n} \nu b_{\nu} \rightarrow(2 / \pi) f(+0)
$$

more generally if (cf. Szász [3, Lemma 6]) 


$$
2 f_{1}(\theta)=(2 / \theta) \int_{0}^{\theta} f(t) d t \rightarrow j
$$

and

$$
\lim _{\delta \downarrow 0} \liminf _{n \rightarrow \infty} \min _{0<\kappa<\delta_{n}} \sum_{n}^{n+k} b_{\nu} \geqq 0,
$$

then

$$
(1 / n) \sum_{1}^{n} \nu b_{\nu} \rightarrow j / \pi
$$

Hence, applying Theorem 2 we have

$$
s_{n}\left(\rho_{n}, \theta_{n}\right) \rightarrow(j / \pi) \sigma(\beta, \gamma), \quad \text { as } n\left(\rho_{n}-1\right) \rightarrow \gamma \text { and } n \theta_{n} \rightarrow \beta ;
$$

$j$ is the generalized jump of $f(\theta)$ at $\theta=0$. For $\gamma=0$ this yields a generalization of formula (1.4). Note that

$$
f_{1}(\theta)=\theta^{-1} \int_{0}^{\theta} f(t) d t=\sum_{1}^{\infty} b_{\nu} \frac{1-\cos \nu \theta}{\nu \theta}=(\theta / 2) \sum_{1}^{\infty} \nu b_{\nu}\left(\frac{\sin (\nu \theta / 2)}{\nu \theta / 2}\right)^{2} ;
$$

$(2 \theta / \pi)\left\{s_{0} / 2+\sum_{1}^{\infty}((\sin \nu \theta) / \nu \theta)^{2} s_{\nu}\right\}$ is called the Riemannian mean of the second kind corresponding to the sequence $\left\{s_{n}\right\}$. It is a regular transform, as is seen from the identity

$$
\frac{2 \theta}{\pi}\left\{1 / 2+\sum_{1}^{\infty}\left(\frac{\sin \nu \theta}{\nu \theta}\right)^{2}\right\}=1
$$

If we assume only that $(C, 2) \lim n b_{n}=j / \pi$ exists, then Theorem 3 yields again a Gibbs' phenomenon in the case $\left(\mathrm{a}^{\prime \prime \prime}\right)$ and $\lambda>0$.

In this connection we introduce two lemmas.

LEMMA 1. If

$$
(1-r) \sum_{1}^{\infty} \tau_{n} r^{n} \rightarrow \tau
$$

and

$$
\tau_{n}^{\prime}=\sum_{1}^{n} \tau_{\nu}>-p n,
$$

for some $p>0$, and all $n>0$, then

$$
(C, 2) \lim \tau_{n}=\tau \text {. }
$$

We have from (4.3) 
hence

$$
(1-r)^{2} \sum_{1}^{\infty}\left(\tau_{n}^{\prime}+p n\right) r^{n} \rightarrow \tau+p \quad \text { as } r \uparrow 1 \text {; }
$$

in view of (4.4) a theorem of Hardy and Littlewood yields

or

$$
\sum_{1}^{n}\left(\tau_{\nu}^{\prime}+p \nu\right) \sim(1 / 2)(\tau+p) n^{2}
$$

which is (4.5).

$$
\sum_{1}^{n} \tau_{\nu}^{\prime} \sim(1 / 2) \tau n^{2}, \quad \text { as } n \rightarrow \infty
$$

LEMMA 2. If (4.1) holds, then $(1-r) \sum_{1}^{\infty} n b_{n} r^{n} \rightarrow j / \pi$. [3, Lemma 5].

Combining these two lemmas it is seen that (4.1) and the assumption

$$
\sum_{1}^{n} \nu b_{\nu}>-p n \quad \text { for some } p>0 \text { and all } n>0,
$$

imply $(C, 2) \lim n b_{n}=j / \pi$. With reference to Theorem 3 the assumptions (4.1) and (4.6) again yield a Gibbs' phenomenon.

In closing we remark that the existence of $f(+0)$ implies itself $(C, 2) \lim n b_{n}$ $=(1 / 2) f(+0)$. A more general result will be given elsewhere.

\section{REFERENCES}

1. W. Rogosinski, Ueber den Einfluss einseitiger Eigenschaften einer Funktion auf ihre Fourierreihe, Schriften der Koenigsberger Gelehrten Gesellschaft Naturwissenschaftliche Klasse vol. 3 (1926) pp. 57-98.

2. - Abschnittsverhalten bei trigonometrischen und insbesondere Fourierschen Reihen, Math. Zeit. vol. 41 (1936) pp. 75-136.

3. O. Szász, Convergence properties of Fourier series, Trans. Amer. Math. Soc. vol. 37 (1935) pp. 483-500.

4. - The jump of a function determined by its Fourier coefficients, Duke Math. J. vol. 4 (1938) pp. 401-407.

5. A. Zygmund, Trigonometrical series, 1935.

University of Cincinnati,

Cincinnati, Ohio 